\title{
Electromechanical responses of poly(3-thiopheneacetic acid)/acrylonitrile-butadiene rubbers
}

\author{
P. Thipdech, R. Kunanuruksapong, A. Sirivat ${ }^{*}$ \\ Conductive and Electroactive Polymer Research Unit The Petroleum and Petrochemical College, \\ Chulalongkorn University, Bangkok, 10330, Thailand
}

Received 19 September 2008; accepted in revised form 11 November 2008

\begin{abstract}
Acrylonitrile-butadiene rubber (NBR) and blends of poly(3-thiopheneacetic acid)/ acrylonitrile-butadiene rubber, P3TAA/NBR, were fabricated, and the electrorheological properties, dielectric, and electrical conductivities were investigated. The electrorheological properties were determined under an oscillatory shear mode in a frequency range of 0.1 to $100 \mathrm{rad} / \mathrm{s}$ at various electric field strengths, from 0 to $2 \mathrm{kV} / \mathrm{mm}$, at a fixed $27^{\circ} \mathrm{C}$ to observe the effects of acrylonitrile content $(\mathrm{ACN})$ in the rubber systems and the conductive particle concentration in the blends. For the pure rubber systems, the storage modulus response $\left(\Delta G^{\prime}\right)$ is linearly dependent on its dielectric constant $\left(\varepsilon^{\prime}\right)$, and increases with the ACN content. For the NBR/P3TAA blends, the storage modulus response varies nonlinearly with the dielectric constant. The bending responses of the rubbers and the blends were investigated in a vertical cantilever fixture. For the pure rubber system, the bending angle and the dielectrophoresis force vary linearly with electric field strength. For the blend system, the bending angle and the dielectrophoresis force vary nonlinearly with electric field strength.
\end{abstract}

Keywords: smart polymers, electrorheological properties, dielectric properties, acrylonitrile-butadiene rubber, poly(3-thiopheneacetic acid)

\section{Introduction}

Various materials have been used as artificial muscles: pneumatic actuators, shape memory alloys (SMAs), and electroactive ceramics. However, these materials have several disadvantages. A most recent approach to address these problems is electroactive polymers (EAPs), having attractive characteristics in their ability to emulate the operation of biological muscles with high fracture toughness, large actuation strains, and inherent vibration damping [1]. Dielectric elastomers belong to a type of EAPs capable of producing large strains and quick electromechanical responses, essential properties required in advanced electromechanical and dielectric applications $[1,2]$. The principle operation of a dielectric elastomer can be described by the electrostatic model of actuation as put forth by
Pelrine et al. [2]. The response of the elastomer is directly proportional to the square of the applied electric field and linearly proportional to the dielectric constant.

Acrylonitrile-butadiene rubber (NBR) is one type of elastomer possessing excellent resistance to petroleum products. With increasing the amount of ACN content, the dielectric properties are expected to change; the molecules become more polar along with an increase in electrical permittivity. Moreover, NBR has been known to possess relatively good miscibility with several conductive polymers [3]. One of these conductive polymers is polythiophene. A substituted poly(thiophene) at 3- and/or 4-position of the thiophene ring not only confers processability to poly(thiophene)s but it can also be used to modify their properties [4]. 
The presence of fillers, such as electrically conductive additives, in elastomers has been observed to change their electrical, dielectric, and mechanical properties, along with their morphology $[3,5,6]$. The incorporation of the conductive materials into dielectric elastomers has been widely studied as both magnetorheological (MR) and electrorheological (ER) fluids. Lokander and Stenberg (2003) studied the MR properties of NBR having different acrylonitrile amount as well as blends of NBR with iron particles [7]. Dynamic mechanical properties, under the influence of an electric field, of doped poly(3-hexylthiophene), P3HT, blended with a silicone elastomer have also been investigated [8]. Shiga (1997) reported the ER effect of polymethacrylic acid cobalt(II) salt (PMACo) particles in silicone gel; increase in the elastic modulus induced by an electric field was $4 \mathrm{kPa}$, with a particle volume fraction of more than $25 \%$. As the particles were incorporated into the rubber matrix, interaction between the particles occurred under an electric field [9].

In our previous work, various matrix systems were used to investigate the electrorheological (ER) properties, with emphasis on the effects of electric field strength, particle concentration, and operating temperature. The systems were poly(dimethyl siloxane) (PDMS) networks containing camphorsulfonic acid (CSA)-doped polyaniline (PANI) particles [10]. The ER properties of PDMS gel and PPV/PDMS blends were studied for the effect of particle electrical conductivity [11]. The ER properties of pure crosslinked polyisoprene elastomers, polythiophene/polyisoprene blends, and $\operatorname{poly}(p-$ phenylene) and an acrylic rubber blends were also investigated $[12,13]$.

In this work, we are interested in the electromechanical properties of elastomeric actuator materials - acrylonitrile-butadiene rubber containing a conductive poly(3-thiopheneacetic acid) towards artificial muscle applications. Several NBRs having various amounts of ACN were chosen as matrices because of their polarity towards the incorporated poly(3-thiopheneacetic acid). The effects of the acrylonitrile content in acrylonitrilebutadiene rubber, and the conductive polymer particle concentration, on the electrorheological and dielectric properties of the pure rubbers and the blends are reported.

\section{Experimental}

\subsection{Materials}

\subsubsection{Synthesis of poly(3-thiopheneacetic acid)}

AR grade 3-thiopheneacetic acid, 3TAA (Fluka) was used as the monomer. Anhydrous ferric chloride, $\mathrm{FeCl}_{3}$ (AR grade, Riedel-de Haën), was used as the oxidant. Chloroform, $\mathrm{CHCl}_{3}$ (AR grade, $\mathrm{BDH}$ ), and methanol, $\mathrm{CH}_{3} \mathrm{OH}$ (AR grade, CARLO ERBA) dried over a molecular sieve for 24 hours under a nitrogen atmosphere and then distilled, were used as solvents. Perchloric acid, $\mathrm{HClO}_{4}$ (AR grade, AnalaR) was used as the dopant. Sulfuric acid, $\mathrm{H}_{2} \mathrm{SO}_{4}$, was used to protect the oxidative decomposition of the monomer. Diethyl ether and deionized water were used to extract materials. Sodium hydroxide, $\mathrm{NaOH}$, was used as the hydrolyzing agent.

\subsubsection{Acrylonitrile-butadiene rubbers (NBR)}

Several commercial grades of acrylonitrile-butadiene rubber (NBR) (Nipol NBR ${ }^{\circledR}$ DN101L, Nipol $\mathrm{NBR}^{\circledR}$ DN 2850, Nipol NBR ${ }^{\circledR}$ DN401L (Zeon Advanced Polymix, Thailand; containing 7\% carboxylic group), Krynac ${ }^{\circledR} 3345 \mathrm{~F}$, and $\mathrm{Krynac}^{\circledR}$ X7.50 (Lanxess)) having bound acrylonitrile amounts and specific gravities of $42.3 \%, 1 ; 28 \%$, $0.97 ; 18.5 \%, 0.94 ; 33 \%, 0.97$; and $26.5 \%, 0.99$ respectively, were used as the elastomeric matrices.

\subsection{Polymerization procedure}

\subsubsection{Preparation of 3-thiophene methyl acetate (TMA)}

The reaction was by oxidative-coupling polymerization according to the method of Kim et al. (1999) [14]. A $10.0 \mathrm{~g}$ amount of 3-thiopheneacetic acid was refluxed for 24 hours in $50 \mathrm{ml}$ of dry methanol (with 1 drop of concentrated $\mathrm{H}_{2} \mathrm{SO}_{4}$ in order to protect the oxidative decomposition of the carboxylic acid group of monomer during oxidative-coupling polymerization). The methanol was evaporated, and the residue extracted with fresh diethyl ether. The extract was washed with deionized water, dried with anhydrous $\mathrm{MgSO}_{4}$, and then filtered. The diethyl ether was evaporated from the filtrate by a rotating evaporator and a TMA product was obtained. 


\subsubsection{Synthesis of poly(3-thiophene methyl acetate) (PTMA)}

A solution of $10 \mathrm{mmol}$ of protected monomer in $20 \mathrm{ml}$ of chloroform was added dropwise to a solution of $40 \mathrm{mmol}$ of ferric chloride dissolved in $30 \mathrm{ml}$ of dry chloroform under nitrogen atmosphere. The oxidant-to-monomer molar ratio was $4: 1$ in all cases. The reaction was carefully maintained at $0^{\circ} \mathrm{C}\left( \pm 0.5^{\circ} \mathrm{C}\right)$ for 24 hours. The reaction mixture was precipitated by pouring it into an excess amount of methanol [1 1]. The product was repeatedly washed with methanol and deionized water to obtain the PTMA.

\subsubsection{Synthesis of poly(3-thiopheneacetic acid) (PTAA)}

PTMA was hydrolyzed by adding $0.5 \mathrm{~g}$ of the PTMA to a $50 \mathrm{ml} 2.0 \mathrm{M} \mathrm{NaOH}$ solution and was heated for 24 hours at $100^{\circ} \mathrm{C}$. The mixture was filtered, neutralized, and precipitated with a dilute $\mathrm{HCl}$ solution $(\sim 0.5 \mathrm{M})$ to obtain the polymer product. P3TAA was washed several times with deionized water before vacuum drying at room temperature for 2 days. The final product, PTAA was ground with a mortar and passed through a $38 \mu \mathrm{m}$ sieve to control the particle size.

\subsection{Preparation of pure acrylonitrile- butadiene rubber}

Crude acrylonitrile-butadiene rubber was purified by dissolving it in $\mathrm{CHCl}_{3}$ ( $\mathrm{AR}$ grade, $\mathrm{BDH}$ ) and coagulating it in methanol, and was subsequently dried (Vallim et al., 1999) [6]. The purified rubber was dissolved in $\mathrm{CHCl}_{3}$ to obtain a rubber solution prior to casting into films with a thickness of around $1 \mathrm{~mm}$. Then the films were left in an air atmosphere at $27 \pm 1^{\circ} \mathrm{C}$ overnight, and later placed in a vacuum oven to remove any remaining solvents.

\subsection{Preparation of the PTAA/acrylonitrile-butadiene rubber blends}

The PTAA/acrylonitrile-butadiene rubber mixtrures were prepared by the mechanical blending of doped and undoped synthesized polythiophene at various particle volume concentrations $(5,10,15$,
20 , and $30 \% \mathrm{vol} / \mathrm{vol}$ ) into the acrylonitrile-butadiene rubber. The solutions were magnetically stirred for $\sim 24 \mathrm{hr}$ at $27^{\circ} \mathrm{C}$. Bubbles were removed in atmosphere at room temperature, $27^{\circ} \mathrm{C}$, overnight prior to casting the solution into films. Then they were placed in a vacuum at $27^{\circ} \mathrm{C}$ to remove any remaining solvent.

\subsection{Characterization methods}

A Fourier transform infrared spectrometer (Thermo Nicolet, Nexus $\pm 4 \mathrm{~cm}$ ), covering a wavenumber range of $4000-400 \mathrm{~cm}^{-1}$ and using a deuterated triglycine sulfate detector, was used to characterize the conductive polymer. The samples were prepared by grinding the conductive polymer particles with $\mathrm{KBr}$ (Carlo Erba Reagent) at a ratio of P3TAA: $\mathrm{KBr}=1: 20$. Then the mixtures were compressed into pellets. The acrylonitrile-butadiene rubber and the polymer blends, were characterized by using a Horizontal Attenuated Total Reflectance accessory (HATR) equipped with $\mathrm{ZnSe}$.

Nuclear Magnetic Resonance, ${ }^{1} \mathrm{H}-\mathrm{NMR}$ (Varian Unity Inova) data were recorded at $25 \pm 1^{\circ} \mathrm{C}$ at a $500 \mathrm{MHz}$ to characterize the synthesized conductive polymer. Deuterated dimethyl sulfoxide was used as the standard solvent.

Particle sizes of the P3TAA powder were determined by using a particle size analyzer (Malvern, Master Sizer X).

A thermogravimetric/differential thermal analyzer (Perkin Elmer, Pyris Diamond), with a temperature scan from 30 to $800^{\circ} \mathrm{C}$ at a heating rate of $10^{\circ} \mathrm{C} / \mathrm{min}$ under $\mathrm{N}_{2}$ atmosphere, was used to investigate the thermal behavior of the synthesized polythiophene, the pure acrylonitrile-butadiene rubber, and the polythiophene/acrylonitrile-butadien rubber blends.

A scanning electron microscope (JOEL, model JSM-5200-2AE) was used to determine the morphological structure of the conductive polymers and the polymer blend morphology. The samples, in pellet form, were cut into small pieces and adhered to a brass-stub by using adhesive tape. Then they were coated with a thin layer of gold by using a JFC-1100E ion-sputtering device prior to observation.

A custom-built two-point probe electrometer (Keithley, Model 6517A) was used to determine the electrical conductivity of the conductive poly- 
mer $(25 \mathrm{~mm}$ diameter and $0.2 \mathrm{~mm}$ thickness prepared by molding with a hydraulic press). The relation $\sigma=(1 / R t)(1 / K)=(I / V t)(1 / K)$ was used to calculate the specific conductivity, where $t$ is the pellet thickness, $I$ is current change, $V$ is applied voltage (voltage drop), and $K$ is the geometric correction factor, which is equal to the ratio $w / l$, where $w$ and $l$ are the probe width and the length, respectively. The geometrical correction factor $(K)$ was determined by calibrating the two-point probe with semi-conducting silicon sheets of known resistivity values. These two probes were connected to a voltmeter (Keithley, Model 6517A), which supplied a constant voltage and recorded the resultant current. Electrical conductivity values of several samples were first measured at various applied voltages to identify their linear Ohmic regimes.

A resistivity testing fixture (Keithley, Model 8009) connected to a source meter (Keithley, Model 6517A) was also used to determine the electrical conductivity of the matrix materials and the blended films. It is a constant voltage source and the resultant current was read under the atmospheric pressure, 54-60\% relative humidity, and at 24 to $25^{\circ} \mathrm{C}$. The volume resistivity $\left(\rho_{v}\right)$ of matrices was calculated following ASTM standard D257, $\rho_{v}=K_{v} R / t$, where $K_{v}$ is the effective area of the guarded electrode for the particular electrode arrangement employed $\left(22.9 \mathrm{~cm}^{2}\right), t$ is the pellet thickness, and $R$ is the volume resistance is ohms. The conductivity of the polymer blends and the polymer matrices were calculated by using this relation $\sigma=1 / \rho_{v}=\left(t_{c} \cdot I\right) /(22.9 \cdot V)$, where $t_{c}$ is the pellet thickness, $I$ is the resultant current, and $V$ is the applied voltage (voltage drop).

Dielectric measurements were carried out in the frequency range of $20 \mathrm{~Hz}$ up to $600 \mathrm{kHz}$ under an applied voltage of $1 \mathrm{~V}$ at $27 \pm 0.5^{\circ} \mathrm{C}$ by using an LCR meter (HP, 4284A), which was connected to and controlled by a melt rheometer (Rheometric Scientific, ARES) fitted with a custom-built copper parallel plate fixture (diameter of $25 \mathrm{~mm}$ ). The samples were coated with a silver conductive paint (Electrolube) to prevent air being trapped between the samples and the electrodes.

A melt rheometer (Rheometric Scientific, ARES) was used to measure the electrorheological properties. It was fitted with a custom-built copper parallel plate fixture (diameter of $25 \mathrm{~mm}$ ). A DC voltage was applied with a DC power supply (Instek, GFG
8216A), which can deliver an electric field up to $4 \mathrm{kV}$. A digital multimeter (Tektronix, CDM250)) was used to monitor the voltage input. In these experiments, oscillatory shear strain was applied and the dynamic moduli $\left(G^{\prime}\right.$ and $\left.G^{\prime \prime}\right)$ were measured as functions of frequency $(0.1-100 \mathrm{rad} / \mathrm{s})$ and electric field strength $(0-2 \mathrm{kV} / \mathrm{mm})$ and at $27 \pm 1^{\circ} \mathrm{C}$. Strain sweep tests were first carried out to determine the appropriate strain to measure $G^{\prime}$ and $G^{\prime \prime}$ in the linear viscoelastic regime. The suitable strain was $0.1 \%$ for all samples. Before each measurement, each sample was presheared at a low frequency $(0.04 \mathrm{rad} / \mathrm{s})$ with and without electric field for of $30 \mathrm{~min}$.

Bending measurements were carried out by using a DC power supply (Goldsen, GPS 30035) connected to an amplifier (Gamma High Voltage, UC5-30P) supplying a high DC voltage to copper electrodes (30 mm long, $30 \mathrm{~mm}$ wide, and $1.0 \mathrm{~mm}$ in thickness; the distance between the electrodes is $10 \mathrm{~mm}$ ). The apparatus setup is shown in Figure 6. The specimens were immersed in silicone oil, having a viscosity of $100 \mathrm{cP}$, between the pair of parallel copper electrode plates. The specimen widths were $5 \mathrm{~mm}$, and thicknesses $0.6 \mathrm{~mm}$. All the measurements were carried out at ambient temperature, $27 \pm 1^{\circ} \mathrm{C}$. The bending responses of the specimens were recorded by a video camera, and the data were analyzed by digital imaging software (Scion Image).

\section{Results and discussion}

\subsection{Characterization of poly(3-thiopheneacetic acid), P3TAA and poly(3-thiopheneacetic acid)/acrylonitrile-butadiene rubber, P3TAA/NBR, blends}

The peak in the FT-IR spectrum of P3TAA at $3000-2800 \mathrm{~cm}^{-1}$ can be assigned to the stretching vibration of the $\mathrm{C}-\mathrm{H}$ band on the thiophene ring. The absorption band at $1735-1750 \mathrm{~cm}^{-1}$ is due to the $\mathrm{C}=\mathrm{O}$ stretching vibrations; the peak at $1432 \mathrm{~cm}^{-1}$ is evidence for the thiophene ring stretching vibration, and the peak at 1300$1200 \mathrm{~cm}^{-1}$ is due to the $\mathrm{C}-\mathrm{O}$ the stretching vibration. These peaks confirm the successful oxidative polymerization of 3-thiophene methyl acetate [15]. According to Senadeera [15], the characteristic peaks of P3TAA at $3200-3000 \mathrm{~cm}^{-1}$ can be 
assigned to the stretching vibration of the $\mathrm{C}-\mathrm{H}$ bond on the thiophene ring. The peak at 3000$2800 \mathrm{~cm}^{-1}$ represents the aliphatic $\mathrm{C}-\mathrm{H}$ bonds; at $1700 \mathrm{~cm}^{-1}$, the $\mathrm{C}=\mathrm{O}$ stretching vibration; at $1400 \mathrm{~cm}^{-1}$, the thiophene ring stretching vibration; and at $1300-1200 \mathrm{~cm}^{-1}$, the $\mathrm{C}-\mathrm{O}$ strectching vibration [15]. The most characteristic feature of the spectrum is the extremely broad $\mathrm{O}-\mathrm{H}$ absorption occurring in the region from 3400 to $2400 \mathrm{~cm}^{-1}$ [14].

The P3TAA/NBR blends were characterized by using an FT-IR spectrometer equipped with HATR. As P3TAA particles were added to the NBR matrix, the peak at $3400 \mathrm{~cm}^{-1}$, which represents the -OH group of poly(3-thiopheneacetic acid), can still be detected from the blend spectrum. It should be noted that the peak wavenumber is slightly higher for the blends having higher P3TAA amounts, indicating some interactions between P3TAA and NBR.

The structure of the P3TAA was characterized by ${ }^{1} \mathrm{H}-\mathrm{NMR}$ to verify the successful synthesis of poly (3-thiopheneacetic acid). The ${ }^{1} \mathrm{H}-\mathrm{NMR}$ spectra of the polymers provide data in agreement with the

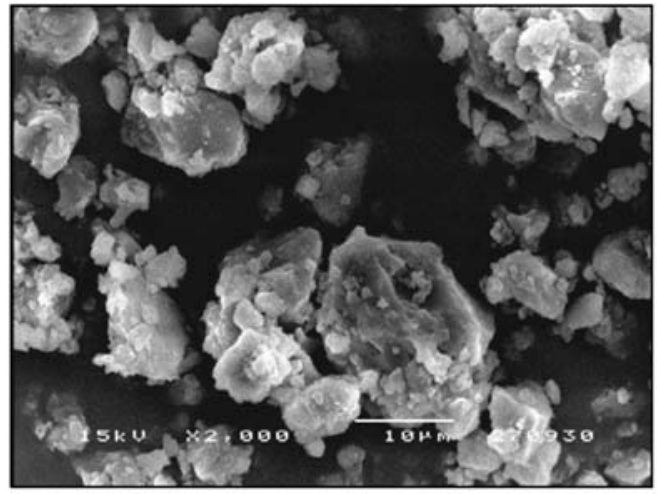

a)

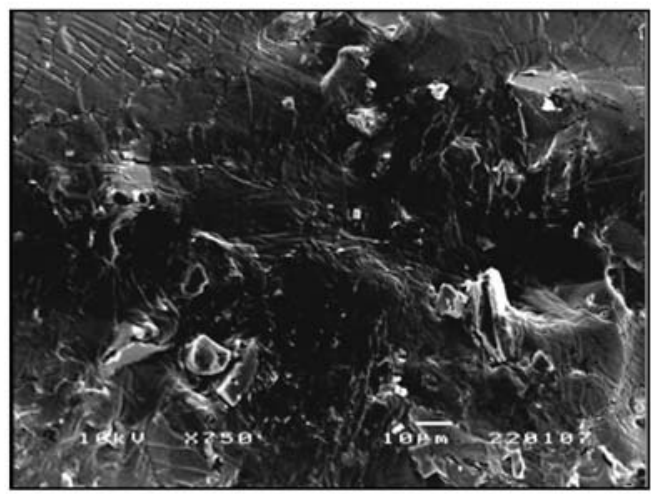

c) expected structures: P3TMA $\delta$ at 7.26-7.3 ppm (m, thiophene ring proton, $1 \mathrm{H}), 3.68 \mathrm{ppm}(\mathrm{s}$, thiophene ring $\left.-\mathrm{CH}_{2-}-2 \mathrm{H}\right)$, and $3.66 \mathrm{ppm}\left(\mathrm{s},-\mathrm{CH}_{3}, 3 \mathrm{H}\right)$; and P3TAA (DMSO) at $12.60 \mathrm{ppm}(\mathrm{s},-\mathrm{COOH}, 1 \mathrm{H})$, 7.55-7.28 ppm (m, thiophene ring proton, $1 \mathrm{H}$ ), and 3.80-3.37 ppm ( $\mathrm{m}$, thiophene ring $-\mathrm{CH}_{2}-, 2 \mathrm{H}$ ). The position at $12.6 \mathrm{ppm}$ is the most important peak of poly(3-thiopheneacetic acid), identifying that the structure changes from poly(3-thiophene methyl acetate) to poly(3-thiopheneacetic acid), since this peak disappears with the presence of poly(3-thiophene methyl acetate) [14].

The TGA thermogram of the undoped P3TAA indicates three transitions: 30-120, 120-300, and 450$650^{\circ} \mathrm{C}$; these refer to the loss of water and residue solvent, the side chain degradation, and the backbone degradation, respectively [16]. Acrylonitrilebutadiene rubber has a better thermal stability, whose main transition temperature starts at $415^{\circ} \mathrm{C}$. The P3TAA/NBR blends were characterized by using a thermogravimetric/differential thermal analyzer under nitrogen gas. The P3TAA/NBR thermogram shows the decomposition temperature at around $230^{\circ} \mathrm{C}$, the decomposition temperature of

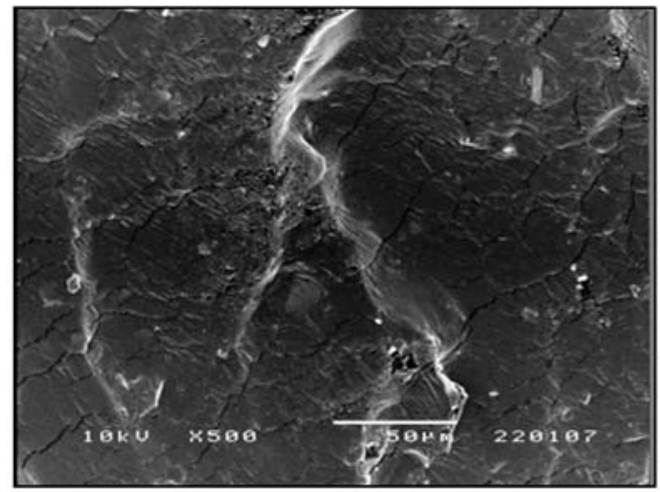

b)

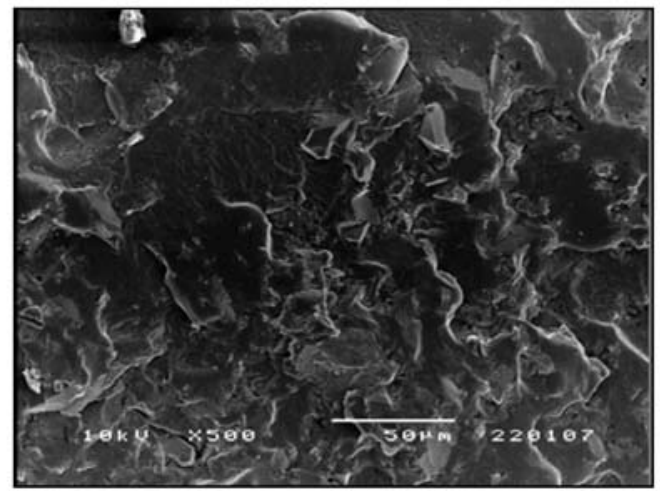

d)

Figure 1. SEM photographs of: (a) undoped poly(3-thiopheneacetic acid) at 2000x; (b) P3TAA_5/NBR1 at 500× (c) P3TAA_20/NBR1 at 500x; and (d) P3TAA_30/NBR1 at 500x 
the P3TAA side chain. As more P3TAA particles are added, the decomposition temperature varies between those of P3TAA and NBR. We infer therefore, that P3TAA enhances the thermal stability of the rubber.

The P3TAA particle size is $20 \mu \mathrm{m}$ with a standard deviation of 0.131 , which it is confirmed by SEM microphotographs (Figure 1a).

The morphology of the blend films was observed from SEM microphotographs to determine the dispersion of the particles. Figures $1 \mathrm{~b}-1 \mathrm{~d}$ show that the P3TAA particles are moderately dispersed in the NBR matrix.

The electrical conductivity of the undoped P3TAA is approximately $5 \cdot 10^{-6} \mathrm{~S} / \mathrm{cm}$ with a standard deviation of $2.73 \cdot 10^{-6} \mathrm{~S} / \mathrm{cm}$. The electrical conductivities of pure NBR is $3.31 \cdot 10^{-10} \mathrm{~S} / \mathrm{cm}$, with a standard deviation of $7.71 \cdot 10^{-11} \mathrm{~S} / \mathrm{cm}$. As more P3TAA particles are added to the NBR matrix, the blend electrical conductivity increases; at the highest concentration $(30 \% \mathrm{vol} / \mathrm{vol})$ it is $4.02 \cdot 10^{-9} \mathrm{~S} / \mathrm{cm}$, with a standard deviation of $8.97 \cdot 10^{-10} \mathrm{~S} / \mathrm{cm}$.

\subsection{Dielectric properties of pure arylonitrile-butadiene rubber and poly(3-thiopheneacetic acid)/pure arylonitrile-butadiene rubber, P3TAA/NBR, blends}

\subsubsection{Effect of acrylonitrile (ACN) content}

The effect of acrylonitrile (ACN) content on the dielectric properties of pure acrylonitrile-butadiene rubbers, NBR1, NBR2, NBR3, NBR4, and NBR5 (with acrylonitrile contents of 42.5, 33, 28, 18.5, and $26.5 \%$, and the last containing the carboxylic group of $7 \%$ ) was investigated in the frequency range of $20 \mathrm{~Hz}$ to $600 \mathrm{kHz}$ at an applied voltage of $1 \mathrm{~V}$ and a fixed temperature of $27^{\circ} \mathrm{C}$. Figure $2 \mathrm{a}$ shows that the dielectric constant $\left(\varepsilon^{\prime}\right)$ of the pure NBRs increases with increasing ACN content. The dielectric constant of NBR having the highest ACN content (NBR1) at a frequency of $20 \mathrm{~Hz}$ is 14 , and the lowest ACN content (NBR4) is 7.8. With increasing frequency $\left(>10^{4} \mathrm{~Hz}\right)$, the dielectric constants decrease; NBR1 shows a higher drop in $\varepsilon^{\prime}$ relative to the others. The results suggest that the increase in $\varepsilon^{\prime}$ is evidently due to the increase in the $\mathrm{C} \equiv \mathrm{N}$ dipoles, leading to an increase in the orientation polarization, which diminishes when the frequency is above $10^{4} \mathrm{~Hz}$ [5].
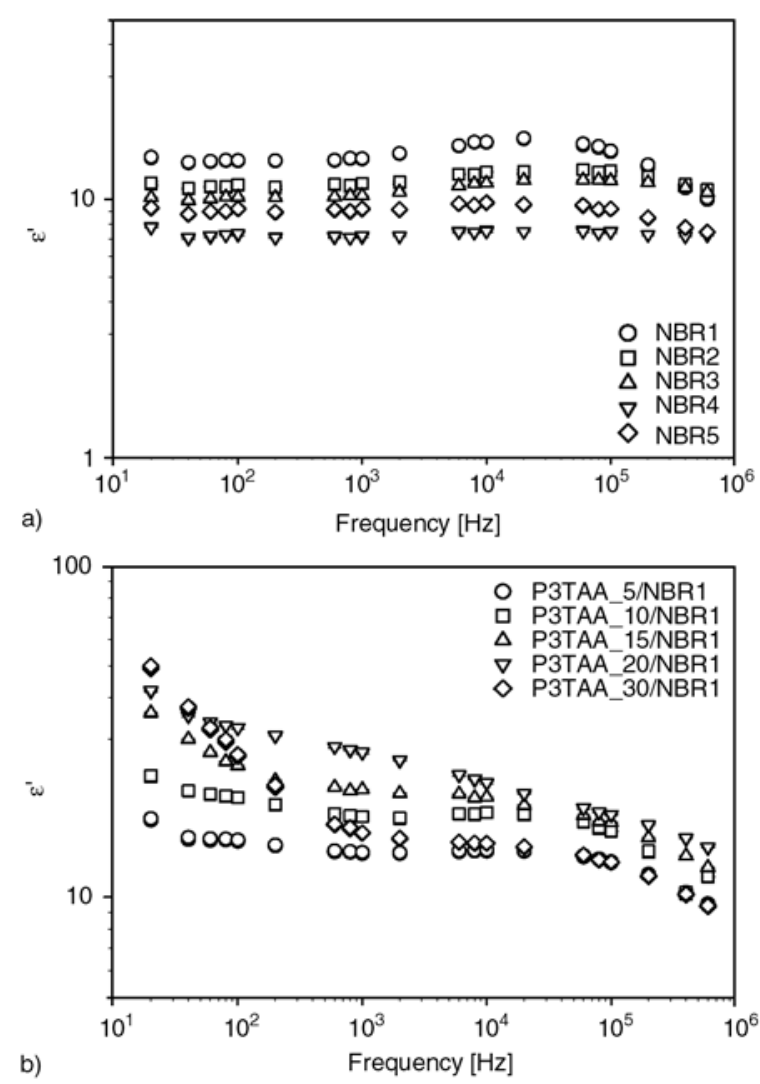

Figure 2. Comparison of the dielectric constants $\left(\varepsilon^{\prime}\right)$ of pure NBR and P3TAA/NBR1 vs. frequency at an applied voltage of $1.0 \mathrm{~V}$, a temperature of $27 \pm 0.5^{\circ} \mathrm{C}$, a gap range of $0.7-1.0 \mathrm{~mm}$ : (a) pure NBR; (b) P3TAA/NBR blends

\subsubsection{Effect of particle concentration}

NBR1 is a matrix containing poly(3-thiopheneacetic acid) at various particle concentrations (5, $10,15,20$, and $30 \%$ vol). In Figure 2b, the dielectric constant increases with increasing particle concentration when the frequency is lower than $10^{2} \mathrm{~Hz}$. The dielectric constant of the blend system having the highest concentration of $30 \% \mathrm{vol} / \mathrm{vol}$ is 48.92 at a frequency of $20 \mathrm{~Hz}$. The increase in dielectric constant with particle concentration occurs as the conductive particles in NBR1 setup the interfacial polarization [17]. From Figure 2b, the dielectric constants of the blend systems monotonically decrease with increasing frequency. In particular, the dielectric constant of the $30 \% \mathrm{vol} / \mathrm{vol}$ system drops sharply, relative to the others, when the frequency is above $10^{2} \mathrm{~Hz}$. This indicates that for the high particle concentration system, the particles lose their ability to be polarized by an electric field due to a screening effect. In addition, the matrix of the $30 \%$ vol system has a smaller number of polar 
sections $(\mathrm{C} \equiv \mathrm{N})$; hence the effect of orientation polarization of the $30 \% \mathrm{vol}$ can be expected to be less than that of the $20 \% \mathrm{vol} / \mathrm{vol}$ system, which inherently contains more NBR sections.

\subsection{Electrorheological properties of pure arylonitrile-butadiene rubber and poly(3-thiopheneacetic acid)/pure arylonitrile-butadiene rubber, P3TAA/NBR, blends}

\subsubsection{Effect of acrylonitrile (ACN) content}

Figures $3 \mathrm{a}$ and $3 \mathrm{~b}$ show the storage modulus responses, defined as $\Delta G^{\prime}=G_{\mathrm{E}}^{\prime}-G_{0}^{\prime}$, where $G_{\mathrm{E}}^{\prime}$ is the storage modulus under electric field, and $G_{0}^{\prime}$ is the storage modulus without electric field, vs. electric field strength. Figure 3a shows the storage modulus responses $\left(\Delta G^{\prime}\right)$ of the pure rubbers increase with electric field strength $(E): \Delta G^{\prime} \propto E^{\alpha}$, where $\alpha$ is the scaling exponent. The $\alpha$ values of pure NBRs having various ACN contents (NBR1, NBR2, NBR3, NBR4, and NBR5) are 0.66, 0.66,
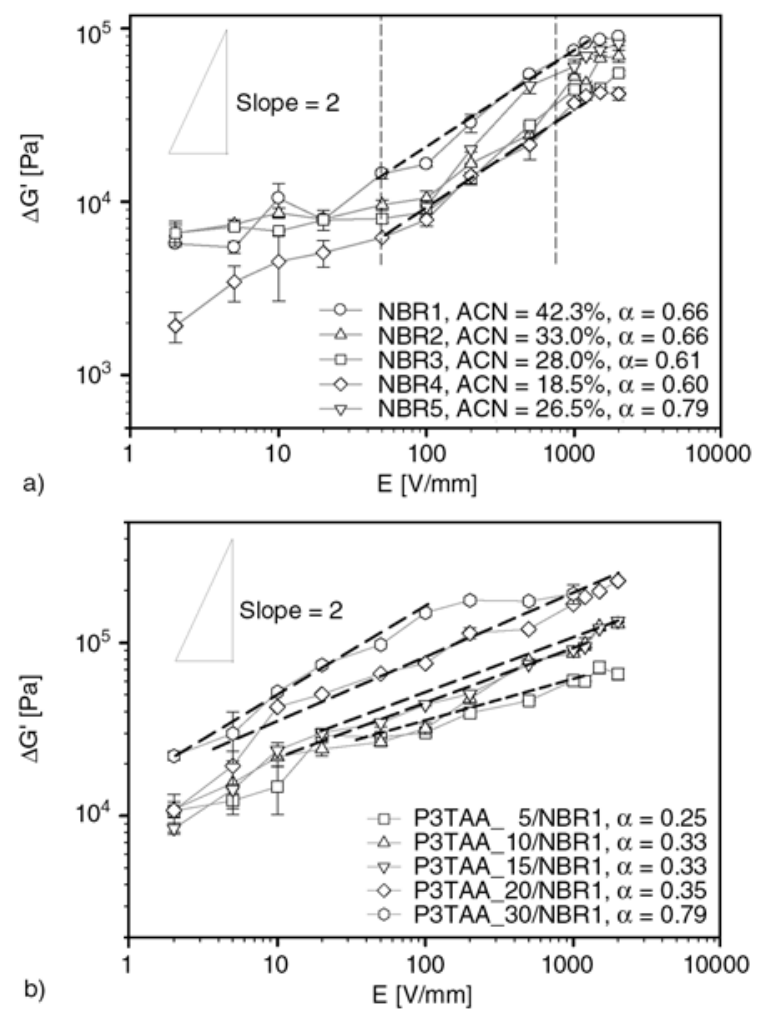

Figure 3. Comparison of the storage modulus responses $\left(\Delta G^{\prime}\right)$ of pure NBR and P3TAA/NBR1 of various particle concentrations $(5,10,15,20$, and $30 \%$ vol) vs. electric filed strength, at a frequency of $1.0 \mathrm{rad} / \mathrm{s}$, a strain of $0.1 \%$, a temperature $27 \pm 0.5^{\circ} \mathrm{C}$, and a gap range of 0.7 to $1.0 \mathrm{~mm}$ : (a) pure NBR; (b) P3TAA/NBR1 blends
$0.61, \quad 0.60$, and 0.79 , respectively, when $0.05 \mathrm{kV} / \mathrm{mm}<E<1.2 \mathrm{kV} / \mathrm{mm}$. The electrostatic model of actuation pressure of Perline [2] predicts that the scaling exponent $\alpha$ should be equal to 2 . In our work, $\Delta G^{\prime}$ inherently depends on ACN content and $\alpha$ varies slightly with $\mathrm{ACN}$ content. As an electric field is applied, electrical dipole moments are generated and electrostatic interactions between the elastomeric segments are induced, leading to an intermolecular interaction acting like an electrical network. The intermolecular interaction results in a loss of chain free movements, higher chain rigidity, and, as a result, higher $G^{\prime}(\omega)$ [18].

\subsubsection{Effect of particle concentration}

P3TAA/NBR1 blends at various P3TAA particle concentrations $(5,10,15,20$, and $30 \% \mathrm{vol} / \mathrm{vol})$ were investigated at electric field strengths between 0 and $2 \mathrm{kV} / \mathrm{mm}$. Figure $3 \mathrm{~b}$ shows the storage modulus $\left(\Delta G^{\prime}\right)$ of each polymer blend system, which generally increases with increasing electric field strength: $\Delta G^{\prime} \propto E^{\alpha}, \alpha$ of each blend system is 0.25 , $0.33,0.33,0.35$, and 0.49 for the particle concentrations of $5,10,15,20$, and $30 \% \mathrm{vol} / \mathrm{vol}$, respectively. The scaling behavior of the storage modulus responses on the electric field strength can be compared to the power law behavior of the MaxwellWagner model [19]: $\Delta G^{\prime} \propto E^{\alpha}$, in which $\alpha$ is predicted to be equal to 2 . For our blend systems, the scaling exponent is less than two. The deviation from the quadratic dependence on electric field at low concentration is because the distances between particles are too large to create a significant particle interaction [21] and the fact that the model is based on the center-to-center distance between the adjacent spheres [19]. At high concentrations, the deviation might also result from the nonlinear conduction proposed by Atten et al. (1994) [20], and the steric hindrance [21]. They suggested that the electric field between particles can become so large that the dielectric breakdown strength of the continuous phase is exceeded.

Figure 4 shows the storage modulus response of P3TAA/NBR1 vs. particle concentration at low electric field strength $(0.1$ and $0.2 \mathrm{kV} / \mathrm{mm})$ and at high electric field strengths (1.0 and $2.0 \mathrm{kV} / \mathrm{mm})$. At low electric field strengths, the response is linear with respect to particle concentration up to $0.1 \% \mathrm{vol} / \mathrm{vol}$; beyond this concentration, the 


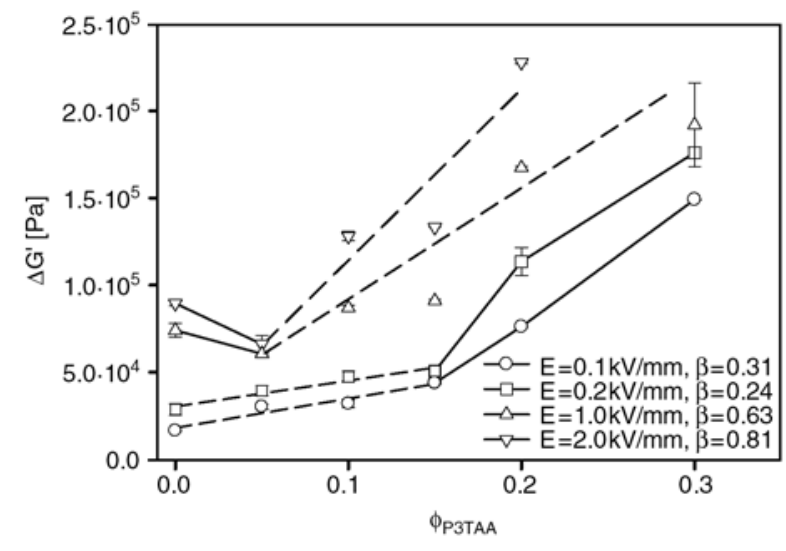

Figure 4. Comparison of the storage modulus responses $\left(\Delta G^{\prime}\right)$ of pure NBR and P3TAA/NBR1 blends at various electric field strengths $(0.1,0.2$, and $1 \mathrm{kV} / \mathrm{mm})$ vs. particle concentration $(\phi=0,5$, $10,15,20$, and $30 \%$ vol) at a frequency of $1.0 \mathrm{rad} / \mathrm{s}$, a strain of $0.1 \%$, a temperature of $27 \pm 0.5^{\circ} \mathrm{C}$, and a gap range of 0.7 to $1.0 \mathrm{~mm}$

response is clearly nonlinear. At high electric field strengths, the response first decreases to a minimum near a particle concentration of $0.5 \% \mathrm{vol} / \mathrm{vol}$; beyond this concentration it appears to increase linearly. At the low electric field strengths, the linear increase in the modulus results from the P3TAA particles behaving as fillers in the matrix. As more P3TAA particles are added, above $15 \% \mathrm{vol} / \mathrm{vol}$, the modulus response sharply increases due to the interparticle interaction. At the high electric field strengths $(1.0,2.0 \mathrm{kV} / \mathrm{mm})$, the initial decrease in the storage modulus response may occur from the cancellation of dipoles of P3TAA particles, which try to align along an electric field, and the particles only act as fillers, which induce an additional free volume, instead of enhancing the storage modulus response [13].

Shiga et al. [8] found a similar effect for $\operatorname{poly}(p-$ phenylene) and silicone elastomer blends. The storage modulus response increased with increasing particle content; it was negligible below $8.4 \%$ vol. Chotpattananont et al. (2004) [16] found that the storage and loss moduli of a polymer blend between polythiophene and silicone oil increased with increasing polythiophene concentration. However, at high concentration, the storage modulus is not responsive to electric field due to the steric hindrance effect. Liu and Shaw (2001) showed that the enhancement of shear modulus of silicone/silica ER elastomers was negligible below $8.0 \%$ vol, but increased dramatically above threshold concentra- tion [21]. At a volume fraction above 55\% vol, the shear modulus decreased because the interparticle force decreased with the steric hindrance effect.

\subsection{Electrorheological properties and dielectric properties of acrylonitrile-butadiene rubber (NBR) and poly(3-thiopheneacetic acid)/acrylonitrile-butadiene rubber (P3TAA/NBR) blends}

\subsubsection{Pure NBR}

In Figure 5a, the storage modulus responses $\left(\Delta G^{\prime}\right)$ at a frequency of $1.0 \mathrm{rad} / \mathrm{s}$ and $0.1 \%$ strain of the pure rubbers increase linearly with increasing the dielectric constant $\varepsilon(20 \mathrm{~Hz})$ or ACN content at both low $(0.1$ and $0.2 \mathrm{kV} / \mathrm{mm})$ and high $(1 \mathrm{kV} / \mathrm{mm})$ electric field strengths. This result is consistent with the electrostatic model of actuation, in which the actuation pressure is proposed to be proportional to the dielectric constant [2].

\subsubsection{Poly(3-thiopheneacetic acid)/ acrylonitrile-butadiene rubber}

P3TAA/NBR blends were fabricated, in which the conductive polymer particles were added to the rubber (NBR1) at $5,10,15,20$, and $30 \% \mathrm{vol} / \mathrm{vol}$, corresponding to P3TAA_5/NBR1, P3TAA_10/ NBR1, P3TAA_15/NBR1, P3TAA_20/NBR1, and P3TAA_30/NBR1, respectively. Figure 5b shows the storage modulus response $\left(\Delta G^{\prime}\right)$ vs. the dielectric constant $\left(\varepsilon^{\prime}\right)$ at $20 \mathrm{~Hz}$, which depends on particle concentration. The storage modulus response $\left(\Delta G^{\prime}\right)$ increases nonlinearly with increasing dielectric constant, notably beyond the particle concentration of $15 \% \mathrm{vol} / \mathrm{vol}$. This result suggests that the interfacial polarization is operative on the $G^{\prime}$ response, as previously shown by Hao et al. (1998) [22]. Referring to Figure $2 b$, it is evident that the $\varepsilon^{\prime}$ of the blends in the Maxwell-Wagner region $(<100 \mathrm{~Hz})$ increases with increasing conductive particle concentration [17]. As more P3TAA particles are added to the NBR, a greater mismatch between their conductivities occurs, resulting in the interfacial polarization. As the conductivity mismatch increases, the interaction between particles is amplified, leading to the greater increase in the storage modulus [22]. 

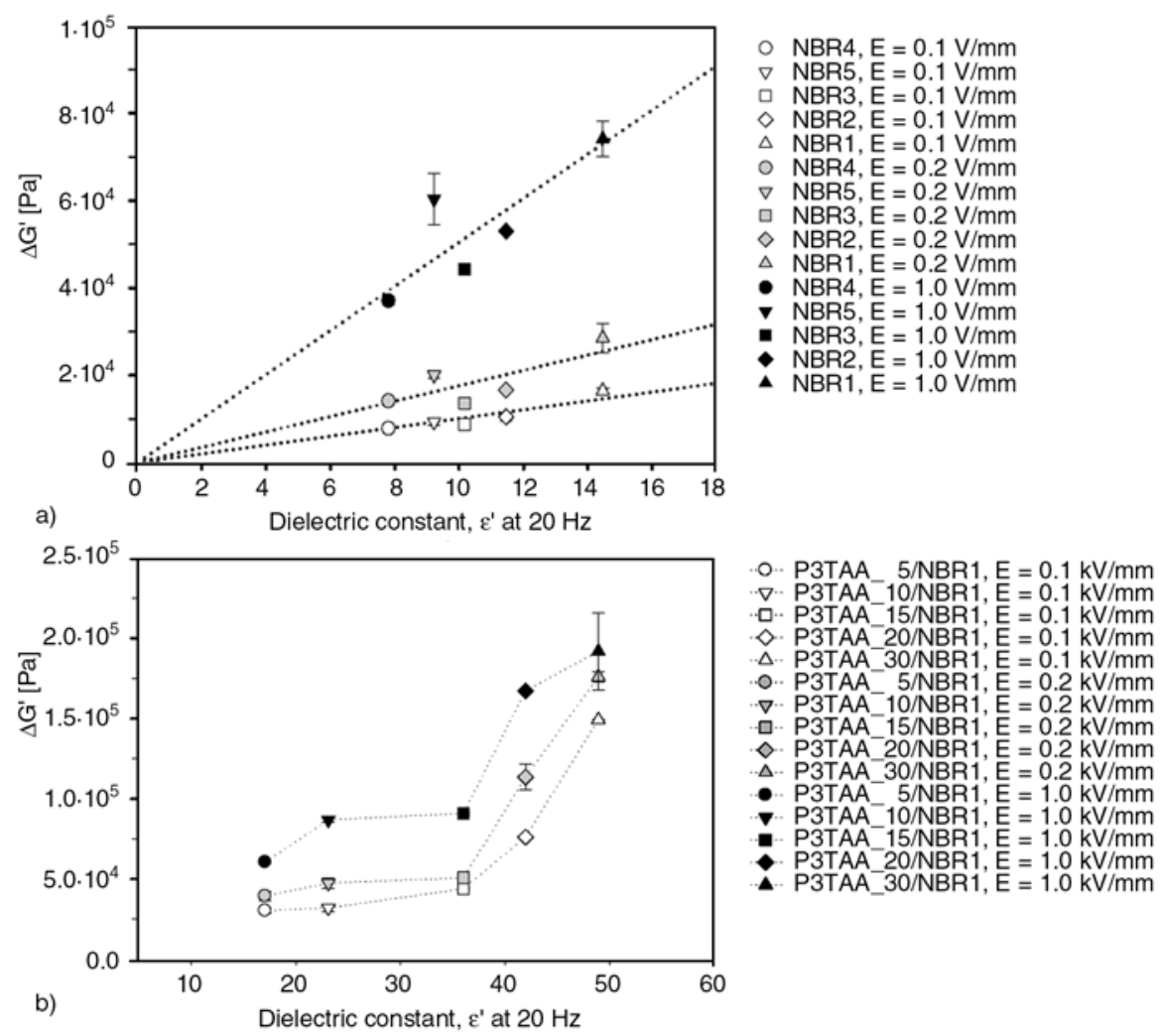

Figure 5. Storage modulus responses at a frequency of $1.0 \mathrm{rad} / \mathrm{s}$, a strain of $0.1 \%$, and at various electric field strengths $(0.1,0.2$, and $1 \mathrm{kV} / \mathrm{mm})$ of pure NBR and P3TAA/ vs. dielectric constant, at $20 \mathrm{~Hz}$, an applied voltage of 1 volt, at $27 \pm 0.5^{\circ} \mathrm{C}$, and at a gap range of 0.7 to $1.0 \mathrm{~mm}$ : (a) pure NBR; (b) P3TAA/NBR1 blends of various particle concentrations $(5,10,15,20$, and $30 \%$ vol $)$

\subsection{Time dependence of the electrorheological response}

The temporal characteristics (the storage modulus) of the pure NBR1 and the P3TAA_20/NBR1 blend, at an electric field strength of $1.2 \mathrm{kV} / \mathrm{mm}$, were investigated. The temporal characteristic of each sample was recorded in the linear viscoelastic regime at a strain of $0.1 \%$ and at a frequency of $1.0 \mathrm{rad} / \mathrm{s}$. Table 1 shows the induction and recovery times of the two samples. They were detected during a time sweep test, in which an electric field was turned on and off alternately. The response of storage modulus, $G^{\prime}$, of the pure NBR system can be divided into two regimes: the initial regime, in which $G^{\prime}$ rapidly overshoots to a large value $\left(\Delta G^{\prime}\right.$ is $23470 \mathrm{~Pa}$ ) on the first cycle, followed by an irreversible decay. The time required for $G^{\prime}$ to reach the steady-state value under electric field is called the induction time, $\tau_{\text {ind }}$. For NBR1, the $\tau_{\text {ind }}$ is $1000 \mathrm{~s}$. The time required for $G^{\prime}$ to decay towards its steady-state value when the electric field is turned off is called the recovery time, $\tau_{\text {rec }}$. It takes longer time to decay to the steady-state $\left(\tau_{\text {rec }}\right.$ is $1500 \mathrm{~s}$ ). However, $G^{\prime}$ does not recover its initial state. The response decreases only by $1460 \mathrm{~Pa}$. As the electric field is turned on again, the response slightly increases until reaching its steady-state. As the electric field is applied to the rubber, the dipoles of the $\mathrm{C} \equiv \mathrm{N}$ segment align themselves along the electric field. When the electric field is turned off, some dipole moments remain due to the interaction in the $\mathrm{C} \equiv \mathrm{N}$, leading to the irreversible storage modulus responses. Berlepsch et al. (1989) [23] studied a stretched copolymer of acrylonitrile and methy-

Table 1. Induction time and recovery time at an electric strength of $1.2 \mathrm{kV} / \mathrm{mm}$ of the pure NBR system and the P3TAA_10/NBR1 blend

\begin{tabular}{|l|c|c|c|c|}
\hline \multicolumn{1}{|c|}{ Sample } & Induction time $\left(\tau_{\text {ind }}\right)[\mathbf{s}]$ & Reduction time $\left(\tau_{\text {rec }}\right)[\mathbf{s}]$ & $\Delta \mathbf{G}_{\text {ind }}[\mathbf{P a}]$ & \multicolumn{1}{c|}{$\Delta \mathbf{G}_{\text {rec }}[\mathbf{P a}]$} \\
\hline NBR1 & 1000 & 1500 & 23470 & 1460 \\
\hline P3TAA_20/NBR1 & 1500 & 3000 & 209920 & 4010 \\
\hline
\end{tabular}


lacrylate. They found that their samples showed appreciable piezoelectric activity [23]. Eid and ElNashar (2006) investigated the relaxation time of polymer blend between NBR and EPDM, they found that the relaxation time increased slightly by the addition of NBR to the EPDM. The relaxation time could be associated with those orientations of small aggregates caused by the movement of the main chain [5].

P3TAA_20/NBR1 is also an irreversible system. Table 1 shows that the modulus response and $\tau_{\text {ind }}$ of the first cycle are $209920 \mathrm{~Pa}$ and $1500 \mathrm{~s}$, respectively; both values are larger than those of the pure NBR1 system. The blend system takes a longer time to decay to the steady-state when the electric field is turned off. This result suggests that there are some irreversible interactions between polythiophene particles, perhaps due to the hydrogen bonding between adjacent polythiophene particles, and to the residual dipole moments inducing permanent interparticle interactions [12].

\subsection{Bending response}

Pure NBR1 and P3TAA_10/NBR1 blend bending was observed in response to electric field. The samples were clamped between copper plates and immersed in silicone oil. Different voltages were

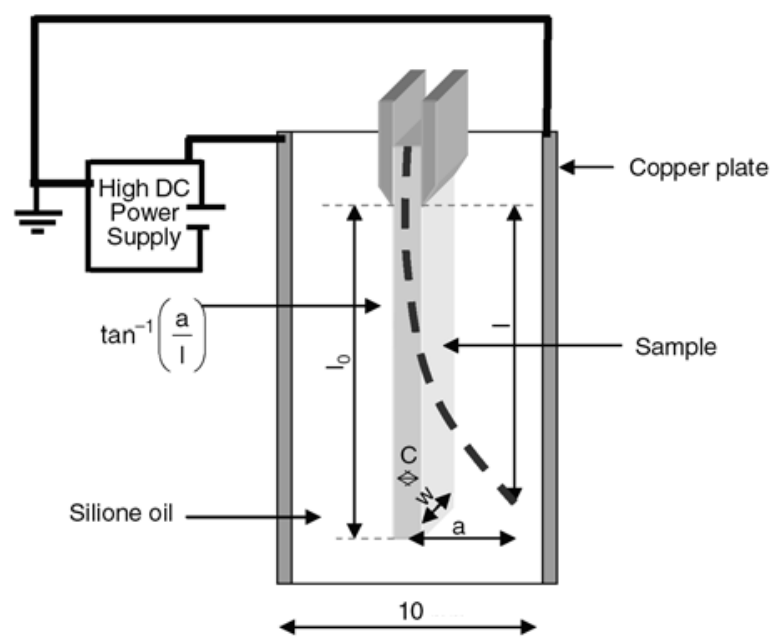

Figure 6. Schematic of the bending response measurement of pure NBR1 and P3TAA_10/NBR1 films suspended vertically in a silicon oil bath and sandwiched between copper plates $(30 \mathrm{~mm}$ long, $30 \mathrm{~mm}$ wide, and $1.0 \mathrm{~mm}$ thick, with a distance of $10 \mathrm{~mm}$ between the electrodes in an acrylic box. A DC electric field is applied horizontally at $\pm 0.5^{\circ} \mathrm{C}$ causing a deflection distance $(a)$ of the film from its original position to a new position (dashed line).

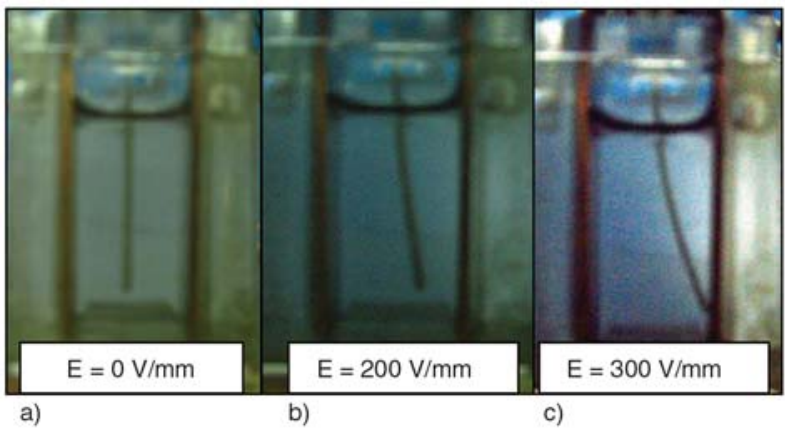

Figure 7. Bending response pictures of pure NBR1 at various applied voltages at $27 \pm 0.5^{\circ} \mathrm{C}$ : (a) $E=$ $0 \mathrm{~V} / \mathrm{mm}$; (b) $E=200 \mathrm{~V} / \mathrm{mm}$; (c) $E=300 \mathrm{~V} / \mathrm{mm}$. Size of pure NBR sample: $0.6 \mathrm{~mm}$ thick, $5.55 \mathrm{~mm}$ in wide, and a weight of $0.1515 \mathrm{~g}$.

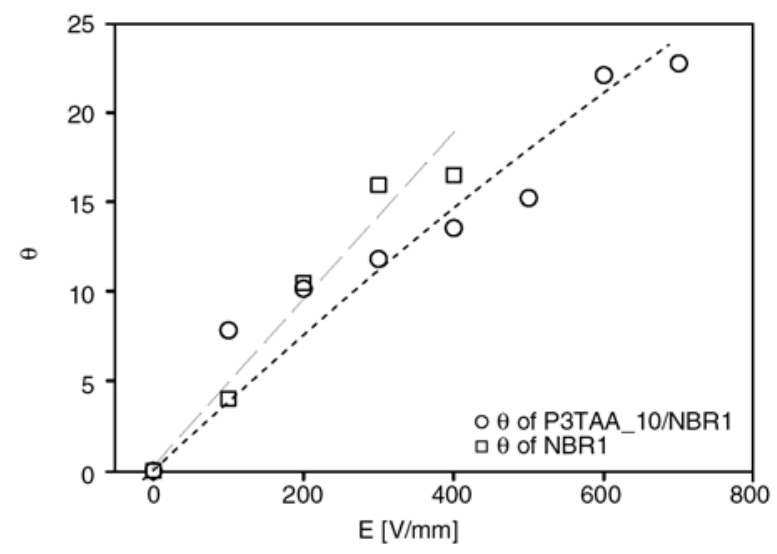

Figure 8. Bending angle of pure NBR1 and P3TAA_10/NBR1 vs. electric field strength at $27 \pm 0.5^{\circ} \mathrm{C}$. The thickness of the pure NBR sample is $0.6 \mathrm{~mm}$ with a weight of $0.1515 \mathrm{~g}$; for the P3TAA_10/NBR1, thickness is $0.5 \mathrm{~mm}$, with a weight of $0.1356 \mathrm{~g}$; both samples have a width of $5.55 \mathrm{~mm}$.

applied to the copper plate (Figure 6). The deflection responses of the samples were recorded by a video camera, as shown in (Figure 7). The bending angle, theta, was calculated from the arctan(theta) obtained from the measured deflection distance $(a)$ and the sample length $(l)$. Figure 8 shows the bending angles of pure NBR1 and the P3TAA/NBR1 blend. For pure NBR1, the bending angle increases linearly with electric field up to $E=400 \mathrm{~V} / \mathrm{mm}$. For the blend system (10\% vol/vol P3TAA), the bending angle increases linearly at low electric field $(<500 \mathrm{~V} / \mathrm{mm})$ and then nonlinearly beyond the electric field strength of $500 \mathrm{~V} / \mathrm{mm}$. However, the bending angle of the blend at low electric field strength $(<200 \mathrm{~V} / \mathrm{mm})$ is comparable to that of the pure system. At higher electric field strength, the bending angle of the blend is clearly lower than that 


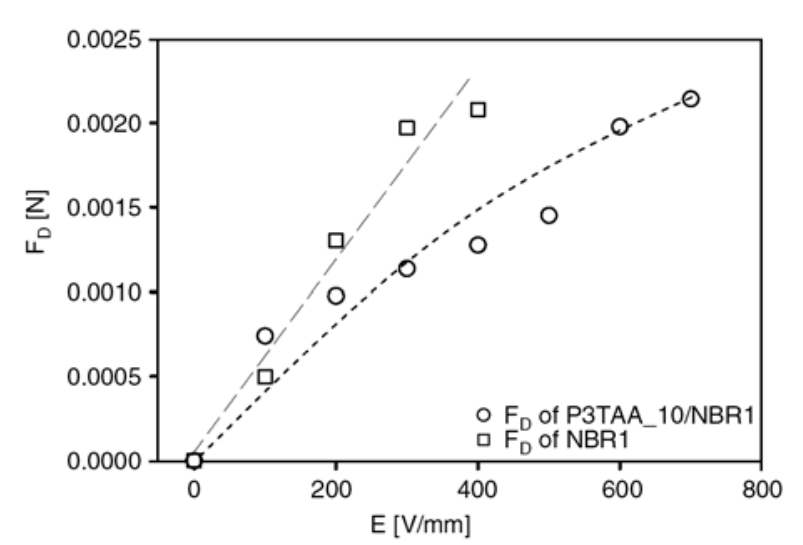

Figure 9. Electric force of pure NBR1 and P3TAA_10/NBR1 vs. electric field strength at $27 \pm 0.5^{\circ} \mathrm{C}$. The thickness of the pure NBR sample is $0.6 \mathrm{~mm}$, with a weight of $0.1515 \mathrm{~g}$; for the P3TAA_10/NBR1, the thickness is $0.5 \mathrm{~mm}$, the weight is $0.1356 \mathrm{~g}$; both samples have a width of $5.55 \mathrm{~mm}$

of the pure rubber system. It may noted that the both pure rubber and the blend bend towards the neutral copper electrode.

Figure 9 shows the resultant dielectrophoresis forces $\left(F_{D}\right)$ calculated from the horizontal force balance Equation (1):

$$
F_{D}=m g \sin \theta+P_{E}(\theta)
$$

where $m$ is the sample mass [kg], $g$ is the gravity $\left(9.8 \mathrm{~m} / \mathrm{s}^{2}\right), \theta$ is the bending angle, and $P_{E}$ is the resisting elastic force $[\mathrm{N}]$ calculated from Equation (2) [24]:

$P_{E}=\frac{3 E I a}{l_{0}^{3}}$

where $E$ is Young's modulus which is equal to $2 G^{\prime}(1+v),-G^{\prime}$ is the shear modulus at a particular electric field, $v$ is Poisson's ratio equal to $1 / 2$ (incompressible material) -, and $I$ is the moment of inertia equal to $2 / 3 C^{3} W$, where $C$ is the sample thickness, and $W$ is the sample width. In Equation (2), $a$ is the deflection distance ( $a$ ), and $l_{0}$ is the initial sample length.

From Figure 9, it can be seen that the dielectrophoresis force of the pure NBR1 system increases linearly with electric field. For the blend system, the resultant dielectrophoresis force varies nonlinearly with applied electric field and it is lower than that of the pure rubber system at any electric field strength. For the pure acrylonitrilebutadiene system, as the electric field is applied, positive dipole moments are generated in the direction of the anode, resulting in a repulsive interaction between the pure rubber and the copper anode. On the other hand, for the blend system, negative dipole moments are generated from the P3TAA particles counteracting the matrix dipole moments, resulting in a smaller dielectrophoresis force at a given electric field strength. Thus, the presence of the conductive polymer filler, P3TAA, can serve to control the overall magnitude of the resultant dielectrophoresis force.

\section{Conclusions}

In this study, the electrorheological properties and dielectric properties of acrylonitrile-butadiene rubber (NBR) and poly(3-thiopheneacetic acid)/acrylonitrile-butadiene rubber blends, P3TAA/NBR, were investigated at electric field strengths varying from 0 to $2 \mathrm{kV} / \mathrm{mm}$. Poly(3-thiophene acetic acid) particles were synthesized via oxidative polymerization.

For the pure NBR system, the storage modulus responses $\left(\Delta G^{\prime}\right)$ increase with increasing electric field strength to nearly one order of magnitude. With increasing ACN content, $\Delta G^{\prime}$ slightly increases. The storage modulus $\left(\Delta G^{\prime}\right)$ in the pure rubber system increases linearly with dielectric constant $\left(\varepsilon^{\prime}\right)$.

For the P3TAA/NBR blend systems, at P3TAA particle concentrations of $5,10,15,20$, and $30 \%$ vol, the storage modulus responses $\left(\Delta G^{\prime}\right)$ increase with particle concentration due to the particle-particle interacting under electric field. The storage modulus response becomes saturated at an electric field strength of $1 \mathrm{kV} / \mathrm{mm}$. Unlike the pure rubber systems, $\Delta G^{\prime}$ is nonlinearly correlated with $\varepsilon^{\prime}$. The storage modulus of the blends increases nonlinearly at high particle concentrations above $15 \%$ vol.

For the temporal response, both systems are irreversible since they have residual dipole moments remaining in the systems when the electric field is turned off.

The bending responses of the pure rubber and the blend systems were observed from a vertical cantilever fixture, and the bending angle and electric force were calculated. In the pure rubber system (NBR1), the response and force increase linearly with electric field. For the blend system, the 
response and the force are nonlinearly correlated with electric field. The P3TAA particles set up dipole moments in the opposite direction to those of the rubber matrix, resulting in smaller dielectrophoresis forces.

\section{Acknowledgements}

The authors would like to acknowledge the Conductive \& Electroactive Polymers Research Unit and KFAS, both of Chulalongkorn University, the Thailand Research Fund (TRF, BRG), the Petroleum Petrochemicals and Advanced Materials Consortium, the Royal Thai Government (Fiscal Year 2551), Zeon Advance Polymix (ZAP), and Lanxess of Thailand.

\section{References}

[1] Bar-Cohen Y.: Electroactive polymer (EAP) actuators as artificial muscles: Reality, potential and challenges. SPIE Press, Bellingham (2004).

[2] Perline R., Kornbluh R., Joseph J., Heydt R., Pei Q., Chiba S.: High-field deformation of elastomeric dielectrics for actuators. Materials Science and Engineering: C, 11, 89-100 (2000).

[3] Yong K. C., Foot P. J. S., Morgan H., Cook S., Tinker A. J.: Conductive poly(butadiene-co-acrylonitrile)polyaniline dodecylbenzenesulfonate [NBR-PAni. DBSA] blends prepared in solution. European Polymer Journal, 42, 1716-1727 (2006).

[4] Li Y., Vamvounis G., Holdcroft S.: Facile functionalization of poly(3-alkylthiophene)s via electrophilic substitution. Macromolecules, 34, 141-143 (2001).

[5] Eid M. A. M., El-Nashar D. E.: Filling effect of silica on electrical and mechanical properties of EPDM/ NBR blends. Polymer-Plastics Technology, 45, 675684 (2006).

[6] Vallim M. R., Felisberti M. I., De Paoli M-A.: Blends of polyaniline with nitrilic rubber. Journal of Applied Polymer Science, 75, 677-684 (2000).

[7] Lokander M., Stenberg B.: Performance of isotropic magnetorheological rubber materials. Polymer Testing, 22, 245-251 (2003).

[8] Shiga T., Okada A., Kurauchi T.: Electroviscoelastic effect of polymer blends consisting of silicone elastomer and semiconducting polymer particles. Macromolecules, 26, 6958-6963 (1993).

[9] Shiga T.: Deformation and viscoelastic behavior of polymer gels in electric fields. Advanced in Polymer Science, 134, 133-163 (1997).

[10] Hiamtup P., Sirivat A.,. Jamieson A. M.: Electromechanical response of a soft and flexible actuator based on polyaniline particles embedded in a crosslinked poly(dimethyl siloxane) network. Materials Science and Engineering: C, 28, 1044-1051 (2008).
[11] Naimlang S., Sirivat A.: Dielectrophoresis force and deflection of electroactive poly(p-phenylene-vinylene)/polydimethylsiloxane blends. Smart Materials and Structures, 17, 035036 p8 (2008).

[12] Puvanatvattana T., Chotpattananont D., Hiamtup P., Niamlang S., Sirivat A., Jamieson A. M.: Electric field induced stress moduli in polythiophene/polyisoprene elastomer blends. Reactive and Functional Polymers, 66, 1575-1588 (2006).

[13] Khunanuruksaphong R., Sirivat A.: Poly(p-phenylene) and acrylic elastomer blends for electroactive application. Materials Science and Engineering, A: Structural Materials, 454-455, 453-460 (2007).

[14] Kim B., Chen L., Gong J., Osada Y.: Titration behavior and spectral transitions of water-soluble polythiophene carboxylic acids. Macromolecules, 32, 3964 3969 (1999).

[15] Senadeera G. K. R.: Microwave-assisted steps in the synthesis of poly(3-thiophenylacetic acid). Current Science, 88, 145-148 (2005).

[16] Chotpattananont D., Sirivat A., Jamieson A. M.: Electrorheological properties of perchloric acid-doped polythiophene suspensions. Colloidaland Polymer Science, 282, 357-365 (2004).

[17] George S., Varughese K. T., Thomas S.: Dielectric properties of isotactic polypropylene/nitrile rubber blends: Effects of blend ratio, filler addition, and dynamic vulcanization. Journal of Applied Polymer Science, 73, 255-270 (1999).

[18] Krause S., Bohon K.: Electromechanical response of electrorheological fluids and poly(dimethylsiloxane) networks. Macromolecules, 34, 7179-7189 (2000).

[19] Parthasarathy M., Klingenberg D. J.: Electrorheology: Mechanisms and models. Materials Science and Engineering: R: Reports, 17, 57-103 (1996).

[20] Atten P., Foulc J-N., Felici N.: A conduction model of the electrorheological effect. International Journal of Modern Physics B, 8, 2731-2745 (1994).

[21] Liu B., Shaw T. M.: Electrorheology of filled silicone elastomers. Journal of Rheology, 45, 64-657 (2001).

[22] Hao T., Kawai A., Ikazaki F.: Mechanism of the electrorheological effect: Evidence from the conductive, dielectric, and surface characteristics of water-free electrorheological fluids. Langmuir, 14, 1256-1262 (1998).

[23] Berlepsch H., Künstler W., Wedel A., Danz R., Gei D.: Piezoelectric activity in a copolymer of acrylonitrile and methylacrylate. IEEE Transactions on Electrical Insulation, 24, 357-362 (1989).

[24] Timoshenko S. P., Goodier J. N.: Theory of elasticity. McGraw-Hill, Auckland (1970). 